\title{
EDITORIAL
}

\section{No-Pharmacological Intervention: Pomegranate Juice for the Management of Hypertension and the Improvement of Cardiovascular Health}

\author{
Konstantinos Tziomalos ${ }^{1}$, Michael Doumas ${ }^{2,3}$ and Vasilios G. Athyros, ${ }^{2, *}$ \\ ${ }^{I}$ First Propedeutic Department of Internal Medicine, Medical School, Aristotle University of Thessaloniki, AHEPA Hos- \\ pital, Thessaloniki, Greece; ${ }^{2}$ Second Propedeutic Department of Internal Medicine, Medical School, Aristotle University \\ of Thessaloniki, Hippokration Hospital, Thessaloniki, Greece; ${ }^{3}$ Veteran Administration Medical Center and George \\ Washington University, Washington, DC, USA
}

Keywords: Hypertension, non-pharmacological treatment, cardiovascular health, life's 7 simple, pomegranate juice.

The concept of ideal cardiovascular (CV) health, with emphasis on the prevention of CV disease (CVD), was included by the American Heart Association (AHA) among its strategic goals for 2020 [1]. This concept was intended to focus mainly on the promotion of a healthy lifestyle and the adoption of a multifactorial intervention with nonpharmacological or pharmacological means, aiming at the prevention or the effective control of CVD risk factors [1]. Ideal $\mathrm{CV}$ health is defined as optimal levels of $3 \mathrm{CVD}$ risk factors [blood pressure (BP), fasting plasma glucose and total cholesterol) and 4 behaviours [body mass index (BMI), smoking, physical activity and healthy diet] [1]. These 7 ideal CV metrics, called life's simple 7, are probably the best available markers of life-time CVD risk [2]. Recent studies have shown that the levels of ideal CV health in the United States to be very low at a community level [3-5] and to be associated with cardiac events [3], stroke [2] and total mortality [6]. A large study was conducted in 5,785 young adults (20-39 years old) from 5 international populations: the Minneapolis Childhood Cohort Study, the Princeton Follow-up Study, the Bogalusa Heart Study, the Cardiovascular Risk in Young Finns Study, and the Childhood Determinants of Adult Health (CDAH) Study; all members of the International Childhood Cardiovascular Cohort (i3C) Consortium [7]. Results of the study showed that ideal CV health, as defined by the AHA, was rare among young participants of the study. An amazingly low (only 1\%) percentage of the participants had all 7 health metrics in the 5,785 young adults participating from all international cohorts [7]. Many of the participants had ideal glucose (73\%), cholesterol (64\%), and were non-smokers (64\%); diet (7\%) was the least common metric for participants from any of the cohorts [7]. The lowest prevalence of a clinical CVD risk factor from the life's 7 simple was BP; this was normal in only $52 \%$ of the

*Address correspondence to this author at the 2nd Popedeutic Department of Internal Medicine, Medical School, Aristotle University of Thessaloniki, Hippokration Hospital, Thessaloniki, Greece. 15 Marmara St, Thessaloniki, 55132, Greece; Tel: +30 2310 892606; Fax: +30 2310 835955;

E-mails: athyros@med.auth.gr or vathyros@gmail.com participants [7]. The National Health and Nutrition Examination Surveys (NHANES) 2003-2008 evaluated the prevalence of the $7 \mathrm{CV}$ health metrics in 14,515 adults [8]. Participants were stratified in young (20-39 years), middle-aged (40-64 years) and elderly ( $\geq 65$ years). Less than $1 \%$ of subjects exhibited ideal CV health for all 7 metrics. Among $\mathrm{CV}$ health behaviors, non-smoking was the most prevalent (60.2-90.4\%), ideal Healthy Diet Score was least prevalent $(0.2-2.6 \%)$ across groups, while ideal BMI (36.5-45.3\%) and ideal physical activity levels (50.2-58.8\%) were higher in young adults compared with middle-aged or elderly [8]. Ideal total cholesterol (23.7-36.2\%), blood pressure (11.9\%$16.3 \%)$, and fasting blood glucose (31.2-42.9\%) were less frequent in older adults compared with young and middleaged adults. The prevalence of poor CV health status was lowest in young age compared with higher middle and older ages [8]. Again, ideal BP was the least prevalent among clinical CVD risk factors [8].

A relevant Chinese study included 91,698 participants (72,826 men, age 18-98 years-old), free of myocardial infarction and stroke at baseline (2006-2007) [2]. The hazard ratios (HR) and 95\% confidence interval (CI) for total stroke according to the adherence to life's 7 simple [0 (reference), 1 , $2,3,4,5$, and 6/7 ideal CV metrics] were: $1,0.92(0.69-$ $1.23), 0.69$ (0.52-0.92), 0.52 (0.39-0.68), 0.38 (0.28-0.51), $0.27(0.18-0.40)$, and $0.24(0.11-0.54)$, respectively ( $\mathrm{p}$ for trend $<0.01$ ), after adjusting for age, sex, education, income and hospital [2]. Similar inverse associations were observed for both ischaemic and haemorrhagic stroke ( $\mathrm{p}$ for trend $<0.01$ ) [2]. In this study, high BP played a major role in stroke incidence [2].

The above data suggest that in the effort of achieving ideal CV health, hypertension should be one of the primary targets, because of its high prevalence in the general population and the low levels of effective control [9]. Moreover, if we can control BP and improve some of the other CV metrics of ideal $\mathrm{CV}$ health, this will have significant additive beneficial effects. 
Non-pharmacological interventions to control hypertension or to augment the effects of antihypertensive drug treatment (thereby potentially reducing the need for more drugs), have been used for a long time [10,11]. The recent (2013) ESH/ESC Guidelines for the management of hypertension [12] suggest: salt restriction to 5-6 g/d, moderation of alcohol consumption to $<20-30 \mathrm{~g} / \mathrm{d}$ of ethanol in men and $<10-20 \mathrm{~g} / \mathrm{d}$ in women, increased consumption of vegetables, fruits, and low-fat dairy products, reduction of BMI to $<25$ $\mathrm{kg} / \mathrm{m}^{2}$ and of waist circumference to $<102 \mathrm{~cm}$ in men and $<88 \mathrm{~cm}$ in women, regular exercise (at least $30 \mathrm{~min}$ of moderate dynamic exercise on 5 to 7 days per week), and advice/support for smoking cessation [12]. Moreover, high quality evidence suggests that increased potassium intake is beneficial in most adults without impaired renal handling of potassium for the prevention or control of elevated BP and prevention of stroke (reduction of incidence by $24 \%$ ), without adverse effects on lipid concentrations, catecholamine concentrations or renal function [13]. However, compliance to long-term lifestyle changes to treat hypertension is very low, and special programs and interventions to improve this adherence may need to consider the existing barriers [14]. A low desire, interest or awareness are commonly reported barriers to salt restriction, changes in diet, weight loss, smoking cessation and alcohol reduction [14]. In contrast, the most common barrier to engaging in physical activity to regulate $\mathrm{BP}$ is time limitations and the challenge of managing a co-existing physical condition/disease; arthritis $(60 \%)$, back problems $(41 \%)$, diabetes $(27 \%)$, CVD or stroke $(27 \%)$, asthma $(23 \%)$ and chronic obstructive pulmonary disease (22\%) [14]. Thus, non-pharmacological interventions for the treatment of hypertension are not successful enough [15].

A number of studies have demonstrated the protective effects of foods rich in polyphenols (fruit, tea, wine and cocoa or chocolate and especially citrus fruit) against several CVD risk factors such as hypertension, low density lipoprotein (LDL) particle oxidation and endothelial dysfunction [1618]. The pomegranate tree (Punica granatum), considered to be originated in the Garden of Eden (modern Mesopotamia and specifically Iran), has been extensively used as a folk medicine in many cultures [19]. Today, it is widely cultivated throughout the Mediterranean region of southern Europe, the Middle East and the Caucasus region, northern and tropical Africa, the Indian subcontinent and the drier parts of Southeast Asia [19]. Introduced into Latin America and California by Spanish settlers in 1769 , pomegranate is also cultivated in parts of California and Arizona. Edible parts of pomegranate fruits (about $50 \%$ of total fruit weight) comprise $80 \%$ juice and $20 \%$ seeds. Fresh juice contains $85 \%$ moisture, $10 \%$ total sugars, $1.5 \%$ pectin, ascorbic acid and polyphenols [19]. Pomegranate fruit is considered as a heart-healthy fruit $[19,20]$. Pomegranate is rich in polyphenolic-type antioxidants, including tannins, anthocyanins and several other types of flavonoids [20,21]. The soluble polyphenol content in pomegranate juice (PJ) normally ranges between 0.2 and $1.0 \%$ depending on the fruit variety, and mainly comprises tannins, ellagic anthocyanins, catechins, and gallic and ellagic acids [20,21]. Despite the previously reported CV health benefits of pomegranate and its high content in polyphenols and flavonoids [20-22], there have been very few studies on the anti-hypertensive effects of PJ [23].
A very recent study included 21 hypertensive patients (aged 30-67 years) [23]. These were assigned to receive either PJ $(150 \mathrm{ml} /$ day in a single occasion between lunch and dinner; $\mathrm{n}$ $=11)$ or the same amount of water $(n=10)$ for a period of 2 weeks. PJ consumption was associated with significant reductions in systolic BP $(\mathrm{p}=0.002)$ and diastolic BP $(p=0.038)$, but not flow mediated dilation (FMD) $(p>0.05)$. Serum levels of vascular cell adhesion molecule 1 (VCAM1) $(p=0.008)$ were significantly reduced by $P J$ while those of E-selectin were elevated $(p=0.039)$. However, no significant PJ effect was observed on serum levels of intercellular adhesion molecule 1 (ICAM-1), high-sensitivity C-reactive protein (hs-CRP), lipid profile, and interleukin-6; this null effect might be due to the short duration of the study [23]. Therefore, consumption of PJ for 2 weeks has effective hypotensive action and may also improve endothelial function. These findings suggest $\mathrm{PJ}$ as a beneficial cardioprotective supplement for hypertensive subjects, with actions extending beyond BP reduction [23]. In an earlier (2001) study in hypertensive patients, the consumption of $\mathrm{PJ}(50 \mathrm{ml}, 1.5 \mathrm{mmol}$ of total polyphenols per day, for 2 weeks) decreased serum angiotensin converting enzyme (ACE) activity by $36 \%$ and systolic BP by 5\% [24]. Similar inhibitory effect (31\%) of PJ on serum ACE activity was also observed in vitro [24]. Since a reduction in serum ACE activity was previously shown to attenuate atherosclerosis, PJ can offer protection against CVD, which could also be related to its inhibitory effect on oxidative stress [24]. Another study demonstrated reduced BP in patients with carotid artery stenosis who had consumed pomegranate juice for 3 years [25]. Ten patients were given PJ for 1 year with 5 patients continuing consumption for up to 3 years. Systolic BP was reduced by $21 \%$ after 1 year of PJ consumption and was not further reduced during the 3 years of PJ use [25]. In this study, serum paraoxonase1 (PON-1) activity was increased by $83 \%$, whereas serum LDL basal oxidative state and LDL susceptibility to copper ion-induced oxidation were both significantly reduced by $90 \%$ and $59 \%$, respectively, after 12 months of PJ consumption [25]. Furthermore, serum levels of antibodies against oxidized LDL were decreased by $19 \%$, and in parallel serum total antioxidant status was increased by $130 \%$ after 1 year of PJ consumption [25]. In the control group, common carotid intima-media thickness (cIMT) increased by $9 \%$ during 1 year, whereas PJ consumption resulted in a significant cIMT reduction, by up to $30 \%$, after 1 year [25]. For all studied parameters, the maximal effects were observed after 1 year of PJ consumption. Further consumption of PJ, for up to 3 years, had no additional beneficial effects on IMT and serum PON-1 activity, whereas serum lipid peroxidation was further reduced by up to $16 \%$ after 3 years of PJ consumption [25]. Total and LDL cholesterol were not significantly affected. Regarding the effect of PJ on triglycerides (TGs), recent data suggest an inhibitory activity of PJ on TGs biosynthesis, which could be attributed to a direct effect of PJ on diacylglycerol acyltransferase 1 (DGAT1) activity [26]. In a larger study in subjects with moderate coronary heart disease risk, PJ consumption $(240 \mathrm{ml} /$ day, $\mathrm{n}=146)$ or a control beverage $(\mathrm{n}=143)$ for up to 18 months had no significant effect on overall cIMT progression rate, but may have slowed cIMT progression in subjects with increased oxidative stress and abnormalities in the TG-rich lipoprotein/ high-density lipoprotein (HDL) axis [27]. Regarding HDL, 
the association of PON-1 with HDL stabilizes this antioxidant enzyme. In high-risk patients, especially those with diabetes, PON-1 dissociates from HDL and, as a consequence, becomes less active [28]. PJ consumption augments PON-11 stability and increases its activity; this could lead to retardation of atherosclerosis development [28], especially PJ from the "wonderful" variety of the fruit [29]. Overall, PJ has at least $20 \%$ greatest antioxidant potency than a variety of other antioxidant beverages, including apple juice, açaí juice, black cherry juice, blueberry juice, cranberry juice, Concord grape juice, orange juice, black tea, green tea and white tea [30]. Similar benefits ofPJ consumption were observed in diabetic patients. Indeed, PJ consumption by diabetic patients resulted in anti-oxidative effects on serum and macrophages without worsening glycemic control [31]. Moreover, arterial stiffness of the common carotid arteries in 73 patients with at least one CVD risk factor that consumed PJ ("wonderful" variety, $240 \mathrm{~mL} / \mathrm{d}$ for 1 year) showed trends to increased elasticity in the PJ-treated group versus the placebo-treated group (who received a beverage of similar caloric content, flavor and color) [19]. However, a single dose of PJ following a high-fat meal had no effect on reflection index, stiffness index or diastolic BP, but lowered postprandial plasma TGs concentrations and suppressed the postprandial increase in systolic BP following the high-fat meal [32].

Beyond hypertension, oxidative stress is also causally related with several CVD risk factors such as diabetes, dyslipidaemia, metabolic syndrome and smoking; oxidative stress has been proved to play a key role in the pathogenesis of atherosclerosis [33]. Oxidized LDL (Ox-LDL) is present in atherosclerotic lesions and in plasma from patients with CVD, and it correlates with the presence of angiographically documented complicated plaques [33], thus identifying patients who are at increased risk for future myocardial infarction, independently of other risk factors [34]. Since PJ contains very potent antioxidants (tannins, anthocyanins), which are also considered potent anti-atherogenic agents, it might attenuate atherosclerosis development by reducing oxidative stress in these patients [34]. Indeed, human plasma obtained from healthy subjects after 2 weeks of PJ consumption (50 $\mathrm{mL} \mathrm{PJ}$ concentrate/day, equivalent to $1.5 \mathrm{mmol}$ total polyphenols) demonstrated a significantly $(\mathrm{p}<0.01)$ decreased susceptibility to free radical-induced lipid peroxidation, in comparison to plasma obtained at baseline prior to PJ consumption initiation, as measured by lipid peroxides formation or by total antioxidant status in serum [31,35]. Very recently, a study evaluated a product a new functional beverage based on a de-alcoholized red wine matrix supplemented by a pomegranate extract. This product is expected to have even more potent antioxidant action [36].

Regarding patients with metabolic syndrome (one of the components of which is hypertension), it has been demonstrated that PJ exerts hypoglycaemic effects by increasing insulin sensitivity, inhibiting $\alpha$-glucosidase, and modulating glucose transporter type-4 function, but also lowers total cholesterol and exerts anti-inflammatory effects through the regulation of peroxisome proliferator-activated receptor pathways [37].

Finally, during the last 15 years, numerous studies reported promising results on the anticarcinogenic, and anti- inflammatory properties of $\mathrm{PJ}$, focusing on treatment and prevention of cancer (mainly prostate), dental conditions, erectile dysfunction, bacterial infections and antibiotic resistance, and ultraviolet radiation-induced skin damage [38].

In conclusion, current data suggest that long-term (at least for 1 year) use of PJ has a beneficial effect on BP, improves endothelial function, reduces arterial stiffness and delays or reverses the progression of atherosclerosis. These effects could result in an improvement in CV and overall health status. Therefore, PJ might be useful as an adjunctive therapy for the management of hypertension on top of other non-pharmacological interventions or drug therapy. The use of PJ might reduce the number of drugs or their doses for patients requiring antihypertensive drug therapy. PJ might be more useful in patients with hypertension and high oxidative burden such as those with diabetes, obesity, metabolic syndrome or who smoke. However, our knowledge on the CV effects of PJ are based on studies with a small number of patients and limitations in their design. Therefore, future long-term well-designed studies with polyphenols-rich foods (alone or in combination), but also with isolated phenolic compounds would provide valuable data to establish public health recommendations on the use of polyphenols for CVD and overall health protection.

\section{CONFLICT OF INTEREST}

This editorial was written independently. The authors did not receive financial or professional help with the preparation of the manuscript. The authors have given talks, attended conferences and participated in advisory boards and trials sponsored by various pharmaceutical companies.

\section{ACKNOWLEDGEMENT}

Declared none.

\section{REFERENCES}

[1] Lloyd-Jones DM, Hong Y, Labarthe D, et al. Defining and setting national goals for cardiovascular health promotion and disease reduction: the American Heart Association's strategic Impact Goal through 2020 and beyond. Circulation 2010; 121: 586-613.

[2] Zhang Q, Zhou Y, Gao X, et al. Ideal Cardiovascular Health Metrics and the Risks of Ischemic and Intracerebral Hemorrhagic Stroke. Stroke 2013 Jul 18. [Epub ahead of print]

[3] Liu K, Daviglus ML, Loria CM, et al. Healthy lifestyle through young adulthood and the presence of low cardiovascular disease risk profile in middle age: the coronary artery risk development in (Young) adults (CARDIA) study. Circulation 2012; 125: 996-1004.

[4] Bambs C, Kip KE, Dinga A, Mulukutla SR, Aiyer AN, Reis SE. Low prevalence of "ideal cardiovascular health" in a communitybased population: the heart strategies concentrating on risk evaluation (Heart SCORE) study. Circulation 2011; 123: 850-7.

[5] Go AS, Mozaffarian D, Roger VL, et al. Heart disease and stroke statistics-2013 update: a report from the American Heart Association. Circulation 2013; 127: e6-e245.

[6] Ford ES, Greenlund KJ, Hong Y. Ideal cardiovascular health and mortality from all causes and diseases of the circulatory system among adults in the United States. Circulation 2012; 125: 987-95.

[7] Oikonen M, Laitinen TT, Magnussen CG, et al. Ideal Cardiovascular Health in Young Adult Populations From the United States, Finland, and Australia and Its Association With cIMT: The International Childhood Cardiovascular Cohort Consortium. J Am Heart Assoc 2013; 2: e000244-52.

[8] Shay CM, Ning H, Allen NB, et al. Status of cardiovascular health in US adults: prevalence estimates from the National Health and 
Nutrition Examination Surveys (NHANES) 2003-2008. Circulation 2012 Jan 3; 125(1): 45-56.

[9] Glynn LG, Murphy AW, Smith SM, Schroeder K, Fahey T. Interventions used to improve control of blood pressure in patients with hypertension. Cochrane Database Syst Rev 2010; 3: CD005182.

[10] Beilin LJ, Puddey IB, Burke V. Lifestyle and hypertension. Am J Hypertens 1999; 12: 934-45.

[11] World Hypertension League. Can non-pharmacological interventions reduce doses of drugs needed for the treatment of hypertension? Bull World Health Organ 1992; 70: 685-90.

[12] Mancia G, Fagard R, Narkiewicz $\mathrm{K}$, et al. 2013 ESH/ESC Guidelines for the management of arterial hypertension: The Task Force for the management of arterial hypertension of the European Society of Hypertension (ESH) and of the European Society of Cardiology (ESC). J Hypertens 2013; 31: 1281-357.

[13] Aburto NJ, Hanson S, Gutierrez H, Hooper L, Elliott P, Cappuccio FP. Effect of increased potassium intake on cardiovascular risk factors and disease: systematic review and meta-analyses. BMJ 2013; 346: f1378-96.

[14] Gee ME, Bienek A, Campbell NR, et al. Prevalence of, and barriers to, preventive lifestyle behaviors in hypertension (from a national survey of Canadians with hypertension). Am J Cardiol 2012; 109: $570-5$.

[15] Lopez L, Cook EF, Horng MS, Hicks LS. Lifestyle modification counseling for hypertensive patients: results from the National Health and Nutrition Examination survey 1999-2004. Am J Hypertens 2009; 22: 325-31.

[16] Hamdy O, Ledbury S, Mullooly C, et al. Lifestyle modification improves endothelial function in obese subjects with the insulin resistance syndrome. Diabetes Care 2003; 26: 2119-25.

[17] Keogh JB, Grieger JA, Noakes M, Clifton PM. Flow mediated dilation is impaired by a high-saturated fat diet but not by a highcarbohydrate diet. Arterioscler Thromb Vasc Biol 2005; 25: 12749.

[18] Wu G, Meininger CJ. Regulation of nitric oxide synthesis by dietary factors. Annu Rev Nutr 2002; 22: 61-86.

[19] Aviram M, Rosenblat M. Pomegranate Protection against Cardiovascular Diseases. Evid Based Complement Alternat Med 2012; 382763-80.

[20] Basu A, Penugonda K. Pomegranate juice: A heart-healthy fruit juice. Nutr Rev 2009; 67:49-56.

[21] de Nigris F, Balestrieri ML, Williams-Ignarro S, et al. The influence of pomegranate fruit extract in comparison to regular pomegranate juice and seed oil on nitric oxide and arterial function in obese Zucker rats. Nitric Oxide 2007; 17: 50-4.

[22] Stowe CB. The effects of pomegranate juice consumption on blood pressure and cardiovascular health. Complement Ther Clin Pract 2011; 17: 113-5.

[23] Asgary S, Sahebkar A, Afshani MR, Keshvari M, Haghjooyjavanmard S, Rafieian-Kopaei M. Clinical Evaluation of Blood Pressure Lowering, Endothelial Function Improving, Hypolipidemic and Anti-Inflammatory Effects of Pomegranate Juice in Hypertensive Subjects. Phytother Res 2013 Mar 21. [Epub ahead of print]
[24] Aviram M, Dornfeld L. Pomegranate juice consumption inhibits serum angiotensin converting enzyme activity and reduces systolic blood pressure. Atherosclerosis 2001; 158: 195-8.

[25] Aviram M, Rosenblat M, Gaitini D, et al. Pomegranate juice consumption for 3 years by patients with carotid artery stenosis reduces common carotid intima-media thickness, blood pressure and LDL oxidation. Clin Nutr 2004; 23: 423-33.

[26] Rosenblat M, Aviram M. Pomegranate juice protects macrophages from triglyceride accumulation: inhibitory effect on DGAT1 activity and on triglyceride biosynthesis. Ann Nutr Metab 2011; 58: 19.

[27] Davidson MH, Maki KC, Dicklin MR, et al. Effects of consumption of pomegranate juice on carotid intima-media thickness in men and women at moderate risk for coronary heart disease. Am J Cardiol 2009; 104: 936-42.

[28] Fuhrman B, Volkova N, Aviram M. Pomegranate juice polyphenols increase recombinant paraoxonase-1 binding to highdensity lipoprotein: studies in vitro and in diabetic patients. Nutrition 2010; 26: 359-66.

[29] Rock W, Rosenblat M, Miller-Lotan R, Levy AP, Elias M, Aviram M. Consumption of wonderful variety pomegranate juice and extract by diabetic patients increases paraoxonase 1 association with high-density lipoprotein and stimulates its catalytic activities. J Agric Food Chem 2008; 56: 8704-13.

[30] Seeram NP, Aviram M, Zhang Y, et al. Comparison of antioxidant potency of commonly consumed polyphenol-rich beverages in the United States. J Agric Food Chem 2008; 56: 1415-22.

[31] Rosenblat M, Hayek T, Aviram M. Anti-oxidative effects of pomegranate juice (PJ) consumption by diabetic patients on serum and on macrophages. Atherosclerosis 2006; 187: 363-71.

[32] Mathew AS, Capel-Williams GM, Berry SE, Hall WL. Acute effects of pomegranate extract on postprandial lipaemia, vascular function and blood pressure. Plant Foods Hum Nutr 2012; 67: 351-7.

[33] Basarici I, Altekin RE, Demir I, et al. Associations of isoprostanesrelated oxidative stress with surrogate subclinical and angiographic measure of atherosclerosis. Coron Artery Dis 2007; 18: 615-20.

[34] Aviram M, Dornfeld L, Kaplan M, et al. Pomegranate juice flavonoids inhibit low-density lipoprotein oxidation and cardiovascular diseases: studies in atherosclerotic mice and in humans. Drugs Exp Clin Res 2002; 28: 49-62.

[35] Aviram M, Dornfeld L, Rosenblat M, et al. Pomegranate juice consumption reduces oxidative stress, atherogenic modifications to LDL, and platelet aggregation: studies in humans and in atherosclerotic apolipoprotein E-deficient mice. Am J Clin Nutr 2000; 71: 1062-76.

[36] Tárrega MA, Varela P, Fromentin E, et al. Specific phenolic compounds and sensory properties of a new dealcoholized red wine with pomegranate (Punica granatum L.) extract. Food Sci Technol Int 2013 Jun 17. [Epub ahead of print]

[37] Medjakovic S, Jungbauer A. Pomegranate: a fruit that ameliorates metabolic syndrome. Food Funct 2013; 4: 19-39.

[38] Jurenka JS. Therapeutic applications of pomegranate (Punica granatum L.): a review. Altern Med Rev 2008; 13:128-44.

Received: August 24, 2013

Revised: August 28, 2013

Accepted: August 28, 2013

(C) Tziomalos et al.; Licensee Bentham Open.

This is an open access article licensed under the terms of the Creative Commons Attribution Non-Commercial License (http://creativecommons.org/licenses/by-nc/3.0/) which permits unrestricted, non-commercial use, distribution and reproduction in any medium, provided the work is properly cited. 